\title{
Initial Analysis of and Predictive Model Development for Weather Reroute Advisory Use
}

\author{
Heather Arneson
}

Aviation Systems Division NASA Ames Research Center

Moffett Field, CA 94035 


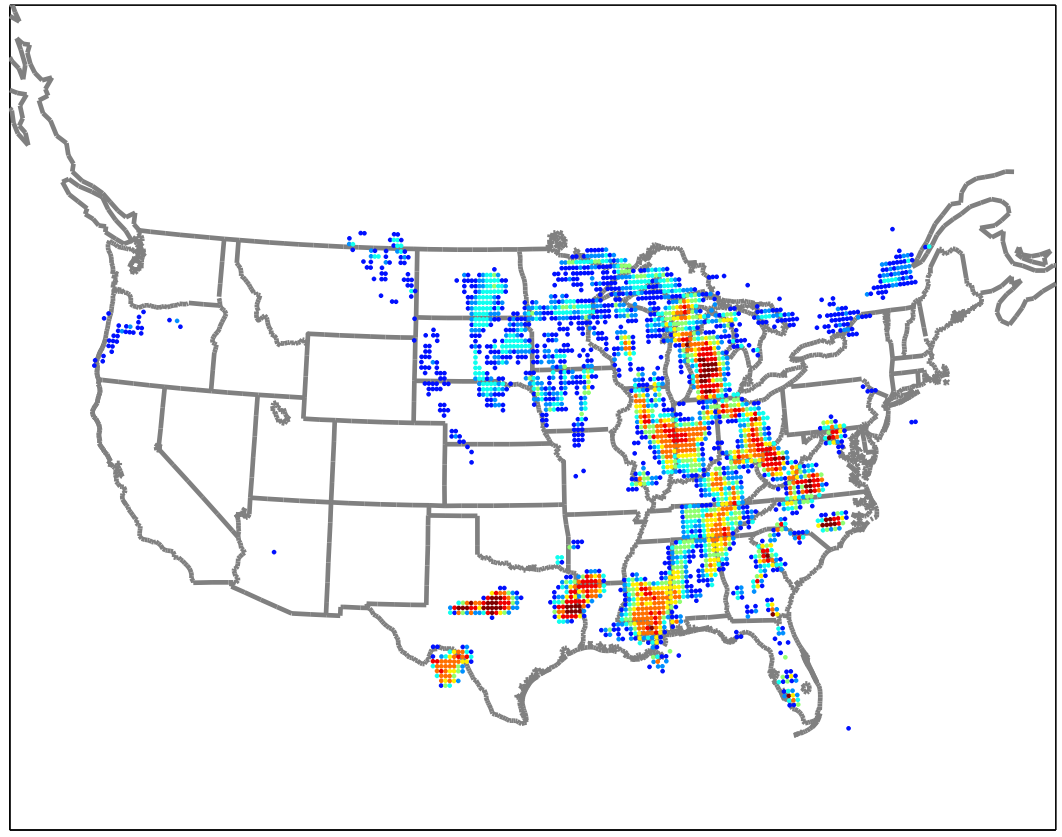




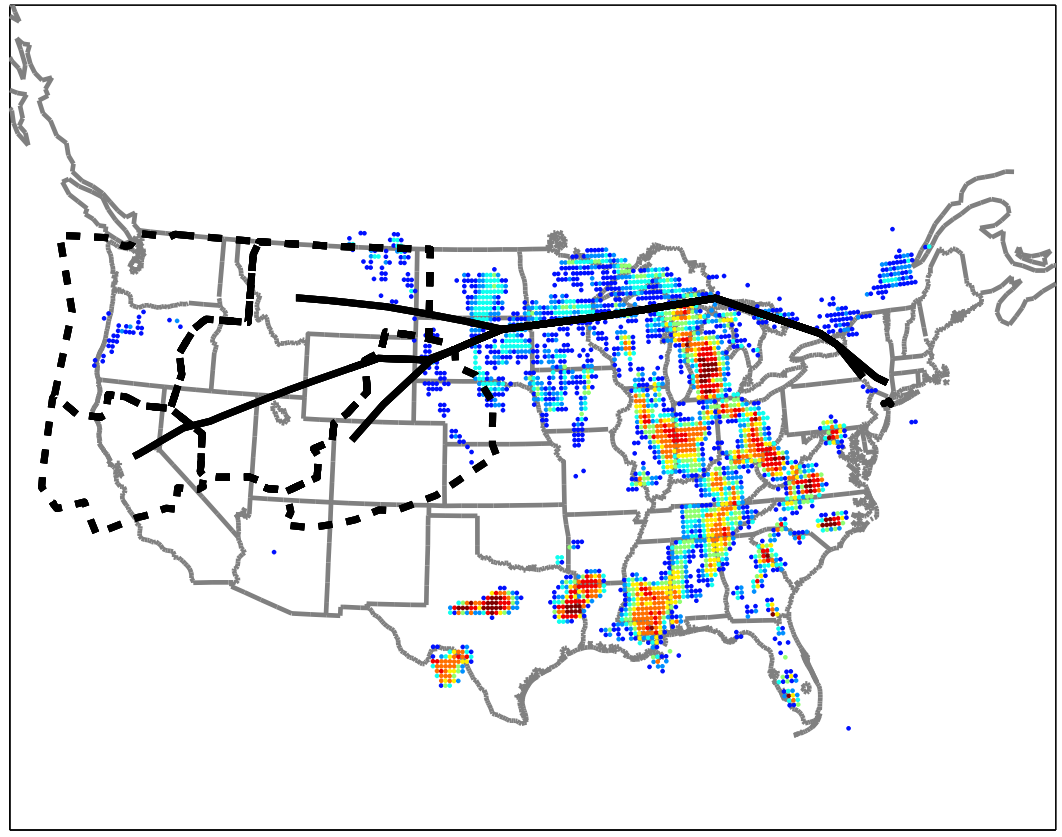




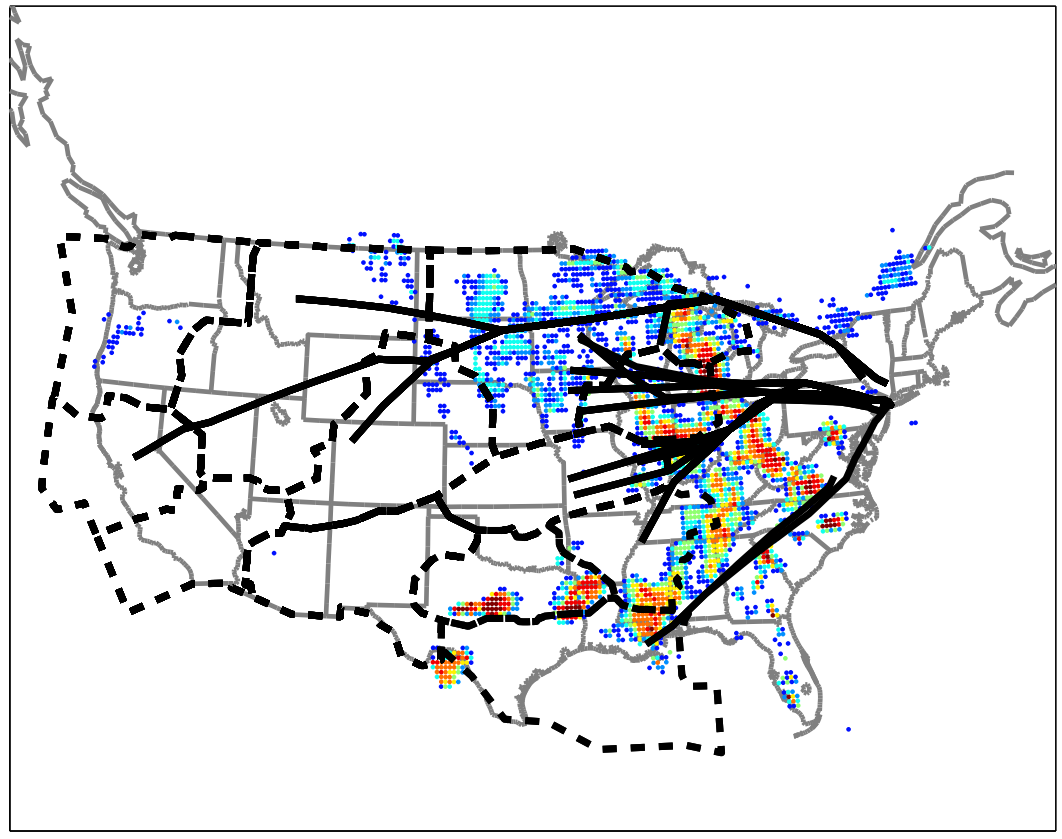


Previous work and ongoing

- Focused on identifying similar weather days

- Analyzing reroutes used on similar days

- Difficult to generate meaningful clusters of days 
Previous work and ongoing

- Focused on identifying similar weather days

- Analyzing reroutes used on similar days

- Difficult to generate meaningful clusters of days

\section{This work}

- Build models to predict the use of reroutes based on weather data 


\section{Objective}

Develop a framework and process to analyze the use of reroutes and develop models to predict reroute use. 


\section{Objective}

Develop a framework and process to analyze the use of reroutes and develop models to predict reroute use.

\section{Challenges}




\section{Objective}

Develop a framework and process to analyze the use of reroutes and develop models to predict reroute use.

\section{Challenges}

- Large amount of weather data available

$\Rightarrow$ difficult to extract relevant features 


\section{Objective}

Develop a framework and process to analyze the use of reroutes and develop models to predict reroute use.

\section{Challenges}

- Large amount of weather data available

$\Rightarrow$ difficult to extract relevant features

- Flexibility in route selection and descriptions

$\Rightarrow$ spatially similar routes with different descriptions 


\section{Objective}

Develop a framework and process to analyze the use of reroutes and develop models to predict reroute use.

\section{Challenges}

- Large amount of weather data available

$\Rightarrow$ difficult to extract relevant features

- Flexibility in route selection and descriptions

$\Rightarrow$ spatially similar routes with different descriptions

- Routes used infrequently

$\Rightarrow$ difficult to find similarities 
- Advisory details

- Methodology

- Identification of routes used by flights

- Identification of similar routes

- Weather feature extraction

- Development of predictive models

- Prediction results

- Concluding remarks 


\section{Outline}

- Advisory details

- Methodology

- Identification of routes used by flights

- Identification of similar routes

- Weather feature extraction

- Development of predictive models

- Prediction results

- Concluding remarks 


\section{Defining advisories}

\section{Advisories consist of ...}

- Name

- Valid time range

- Text description of several routes

- From an origin Center or airport

- To a destination airport 
Advisories consist of . . .

- Name

- Valid time range

- Text description of several routes

- From an origin Center or airport

- To a destination airport

June to August 2011

- 1,669 reroute advisories issued

- 735 unique advisory names

- 34,247 routes

- 2,770 origin-destination pairs 


\section{Example advisory}

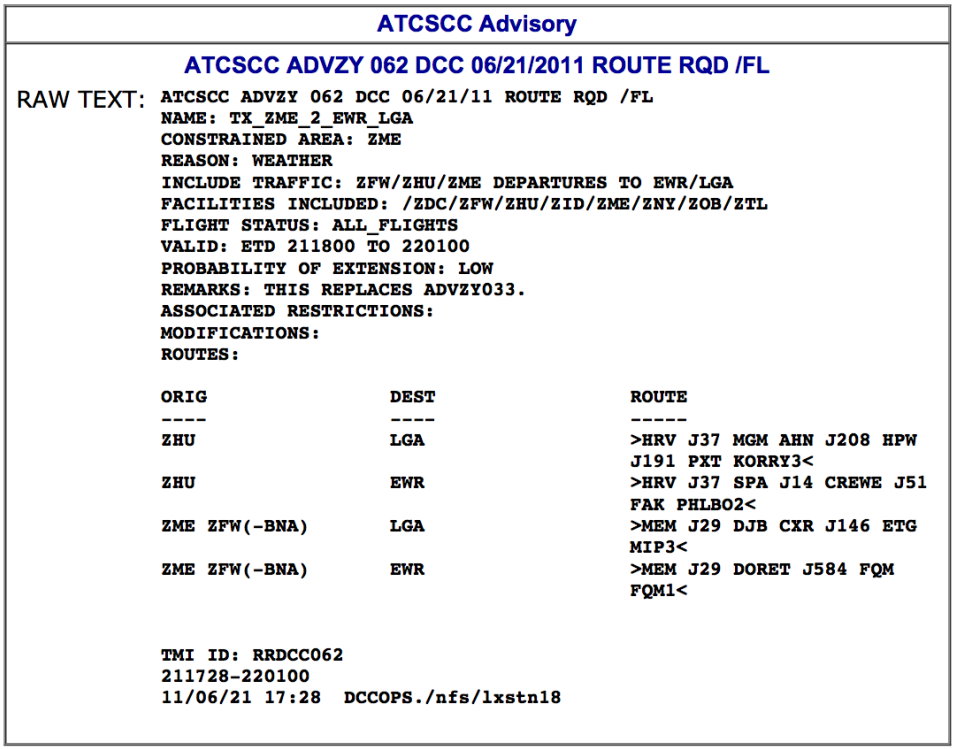




\section{Example advisory}

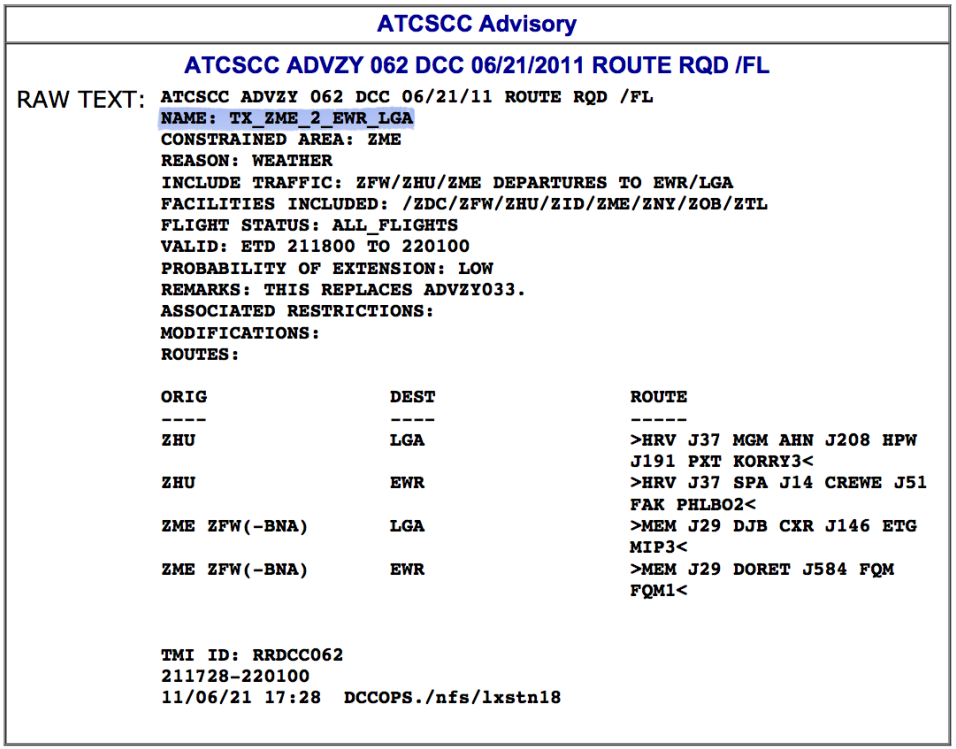




\section{Example advisory}

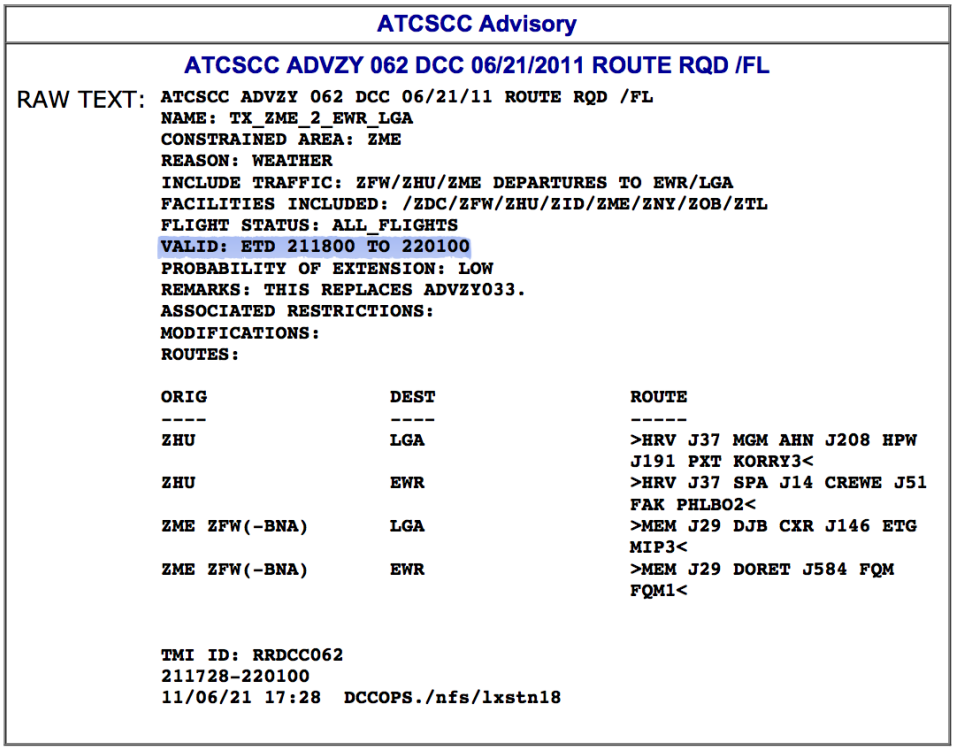




\section{Example advisory}

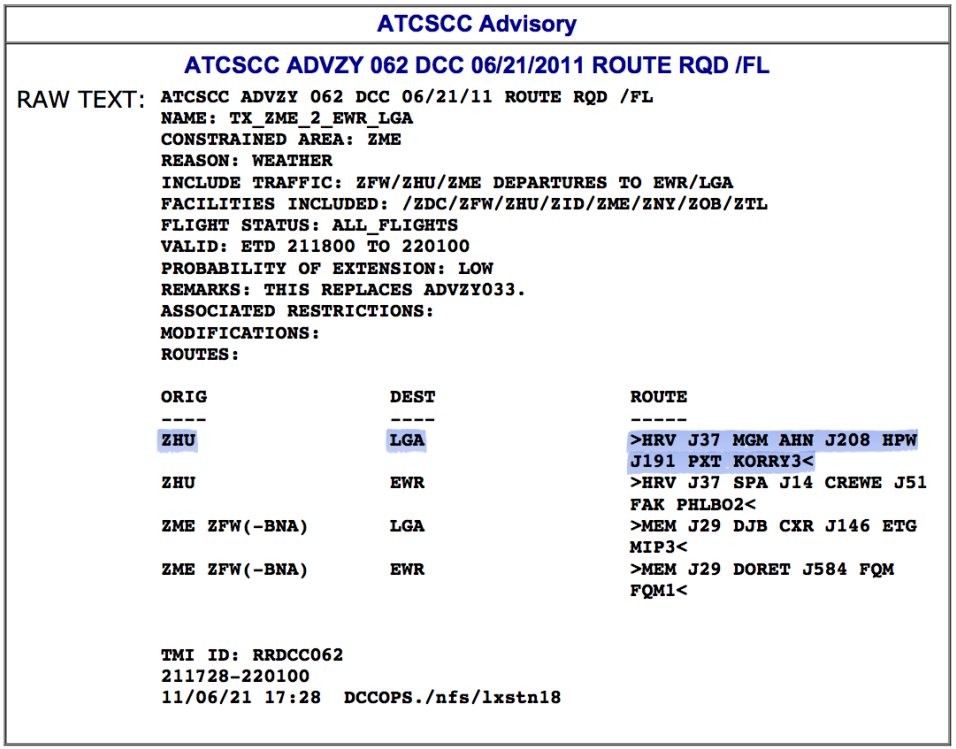




\section{Example advisory}

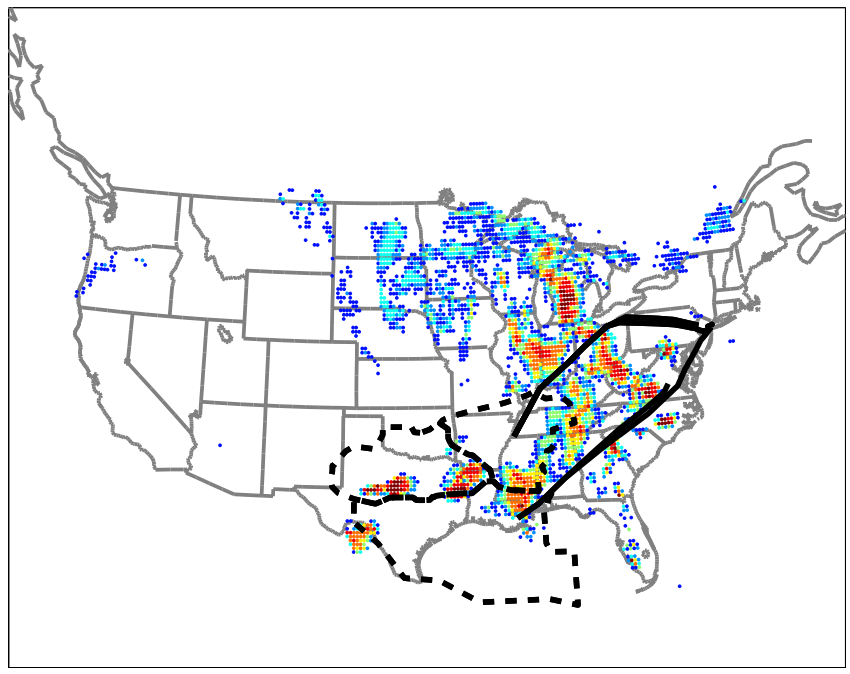




\section{Outline}

- Advisory details

- Methodology

- Identification of routes used by flights

- Identification of similar routes

- Weather feature extraction

- Development of predictive models

- Prediction results

- Concluding remarks 
- Identification of routes used by flights

- Identification of similar routes

- Weather feature extraction

- Development of predictive models 
- Identification of routes used by flights requires distance metric to compare routes and flight tracks

- Identification of similar routes

- Weather feature extraction

- Development of predictive models 
- Identification of routes used by flights requires distance metric to compare routes and flight tracks

- Identification of similar routes requires distance metric to compare routes

- Weather feature extraction

- Development of predictive models 
- Identification of routes used by flights requires distance metric to compare routes and flight tracks

- Identification of similar routes requires distance metric to compare routes

- Weather feature extraction requires domain knowledge

- Development of predictive models 


\section{Outline}

- Methodology

- Identification of routes used by flights

- Weather feature extraction

- Development of predictive models

- Prediction results

- Concluding remarks 


\section{Distance metric}

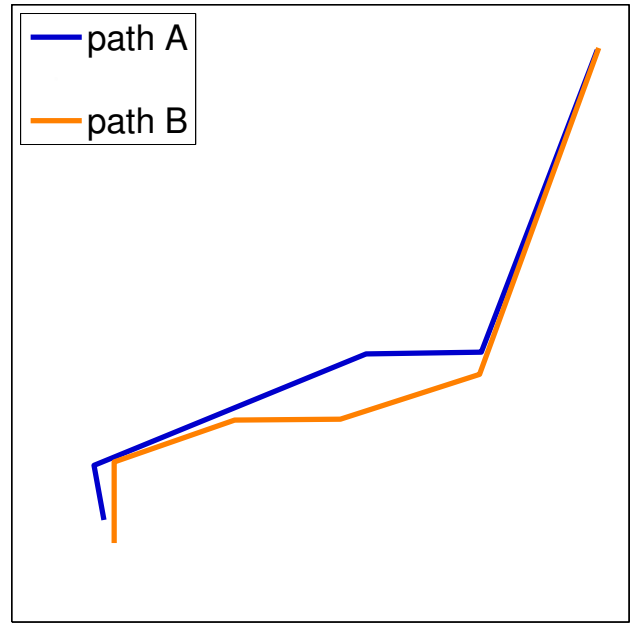




\section{Distance metric}

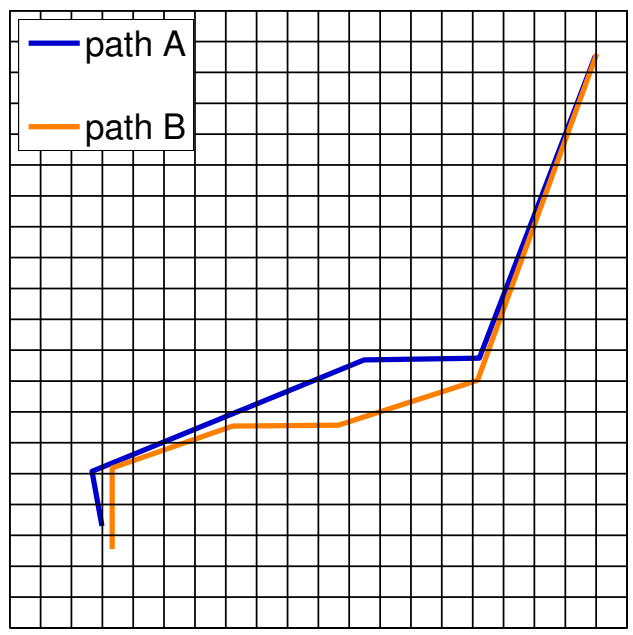




\section{Distance metric}

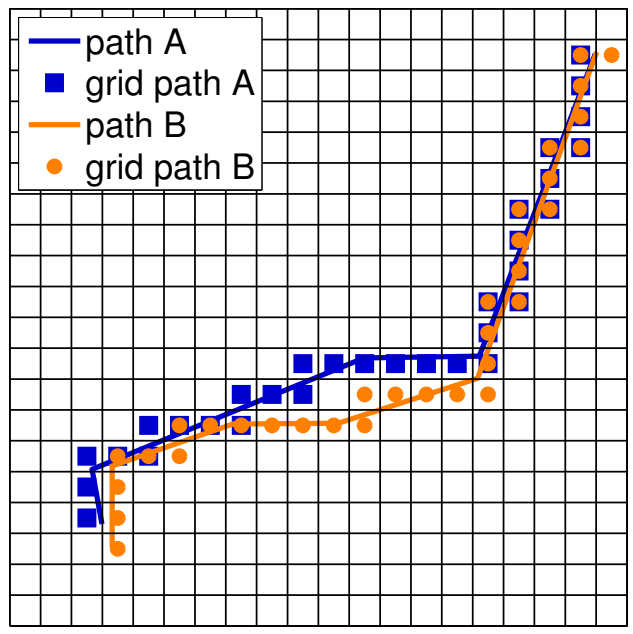




\section{Distance metric}

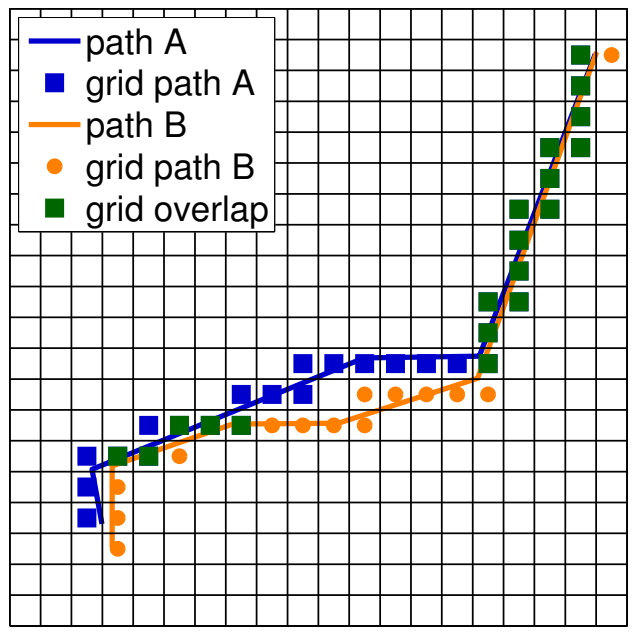




\section{Distance metric}

$\operatorname{distance}($ path $A$, path $B)=1-\frac{\text { length (grid overlap) }}{\min (\text { length (path } A) \text {,length(path } B))}$

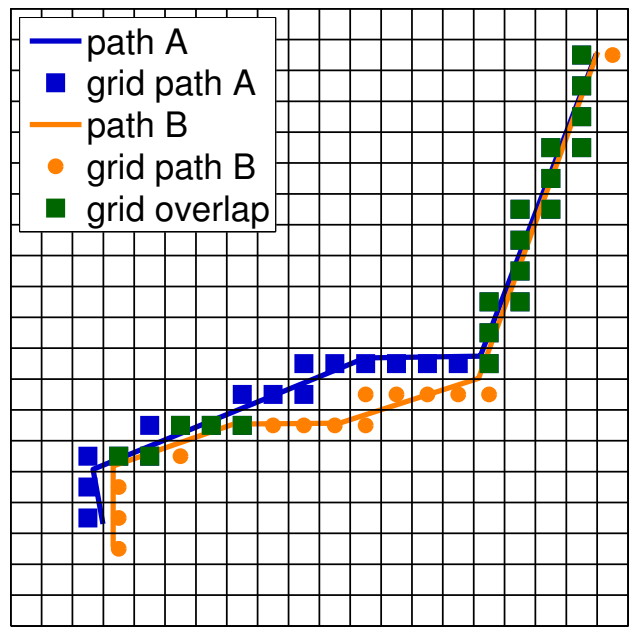




\section{Route usage}

- June through August 2011

- Routes and flights inbound to New York Center (ZNY)

- Define use: flight track and reroute overlap for at least $85 \%$ of shorter path

- Of 4,476 issued routes, 905 were used by at least one flight 


\section{Outline}

- Advisory details

- Methodology

- Identification of similar routes

- Weather feature extraction

- Development of predictive models

- Prediction results

- Concluding remarks 


\section{Cluster routes}

905 used routes grouped into 253 clusters

Example cluster

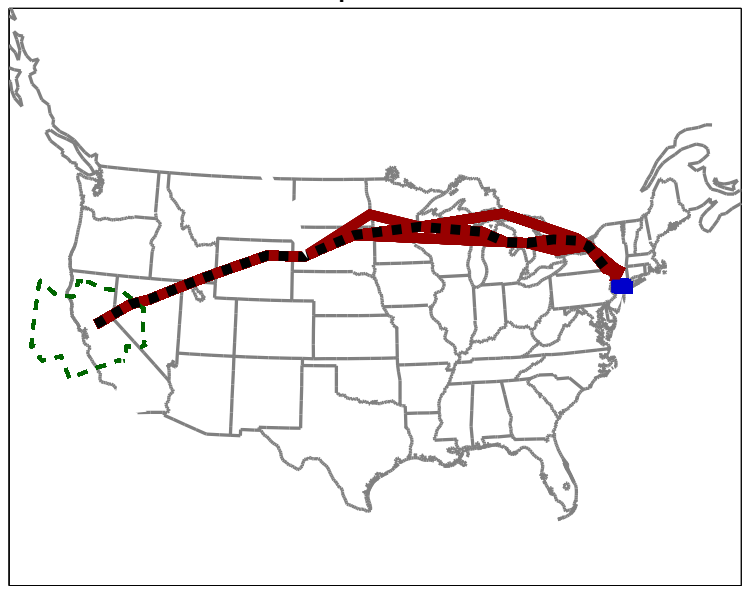




\section{Cluster routes}

905 used routes grouped into 253 clusters

Example cluster

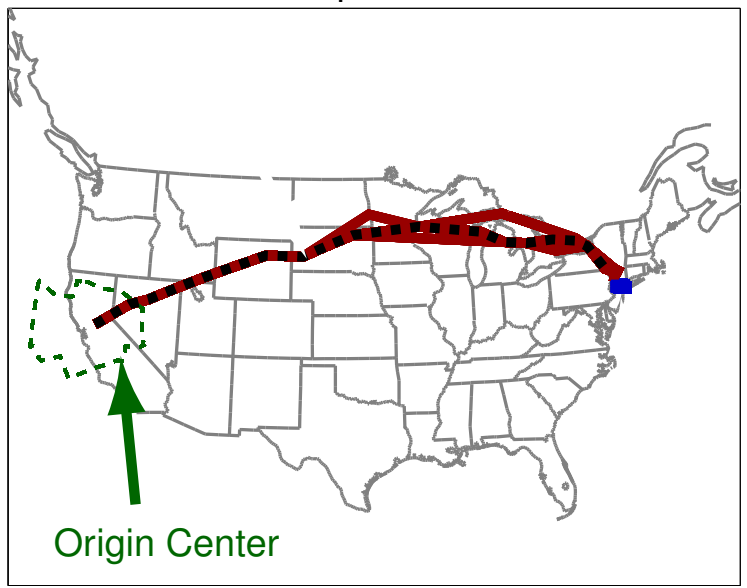




\section{Cluster routes}

905 used routes grouped into 253 clusters

Example cluster

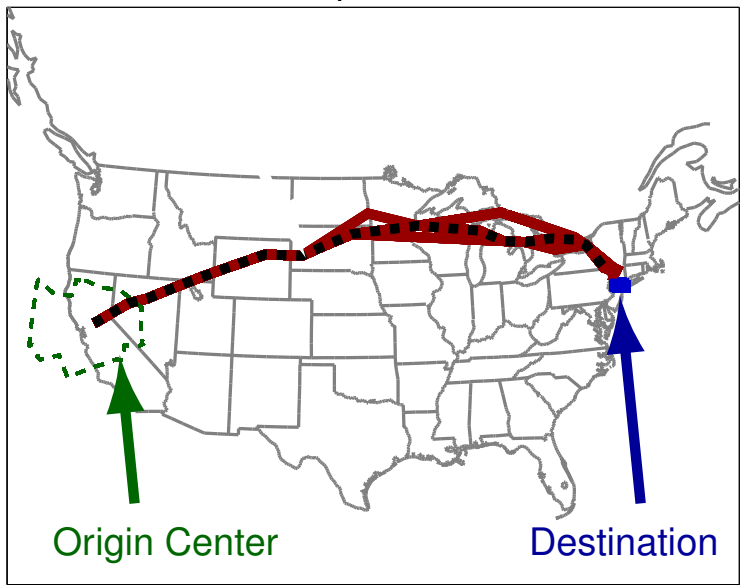




\section{Cluster routes}

905 used routes grouped into 253 clusters

Example cluster

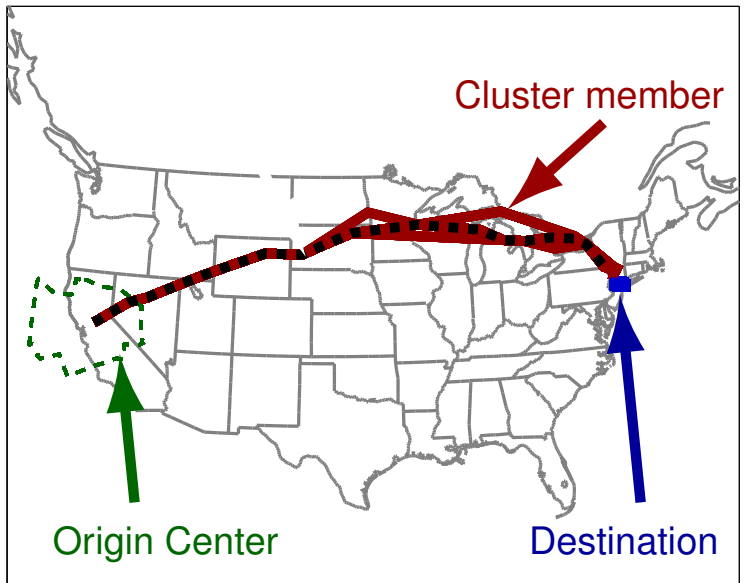




\section{Cluster routes}

905 used routes grouped into 253 clusters

Example cluster

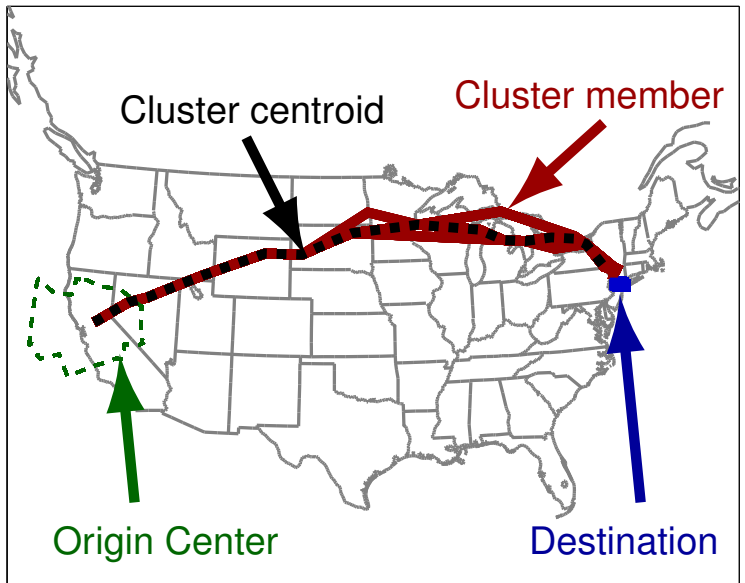




\section{Outline}

- Advisory details

- Methodology

- Identification of routes used by flights

- Identification of similar routes

- Weather feature extraction

- Prediction results

- Concluding remarks 


\section{Convective weather data}

\section{Echo tops}

- Estimates of tops of clouds based on radar measurements

- Values are discrete altitude levels $0 \mathrm{ft}$ to $50,000 \mathrm{ft}$ at $5,000 \mathrm{ft}$ intervals 


\section{Convective weather data}

\section{Echo tops}

- Estimates of tops of clouds based on radar measurements

- Values are discrete altitude levels $0 \mathrm{ft}$ to $50,000 \mathrm{ft}$ at $5,000 \mathrm{ft}$ intervals

- 108,955 data points cover the continental US 


\section{Convective weather data}

\section{Echo tops}

- Estimates of tops of clouds based on radar measurements

- Values are discrete altitude levels $0 \mathrm{ft}$ to $50,000 \mathrm{ft}$ at $5,000 \mathrm{ft}$ intervals

- 108,955 data points cover the continental US

- 2,614,920 echo top values per hour 


\section{Convective weather data}

\section{Echo tops}

- Estimates of tops of clouds based on radar measurements

- Values are discrete altitude levels $0 \mathrm{ft}$ to $50,000 \mathrm{ft}$ at $5,000 \mathrm{ft}$ intervals

- 108,955 data points cover the continental US

- 2,614,920 echo top values per hour

\section{Grid}

- Spatial resolution of $75 \mathrm{nmi}$ by $58 \mathrm{nmi}$

$\left(1.25^{\circ}\right.$ lat by $1.25^{\circ}$ lon $)$

- 1,000 grid elements cover the continental US

- Temporal resolution of one hour

- 1,000 averaged echo top values per hour 


\section{High resolution weather data}

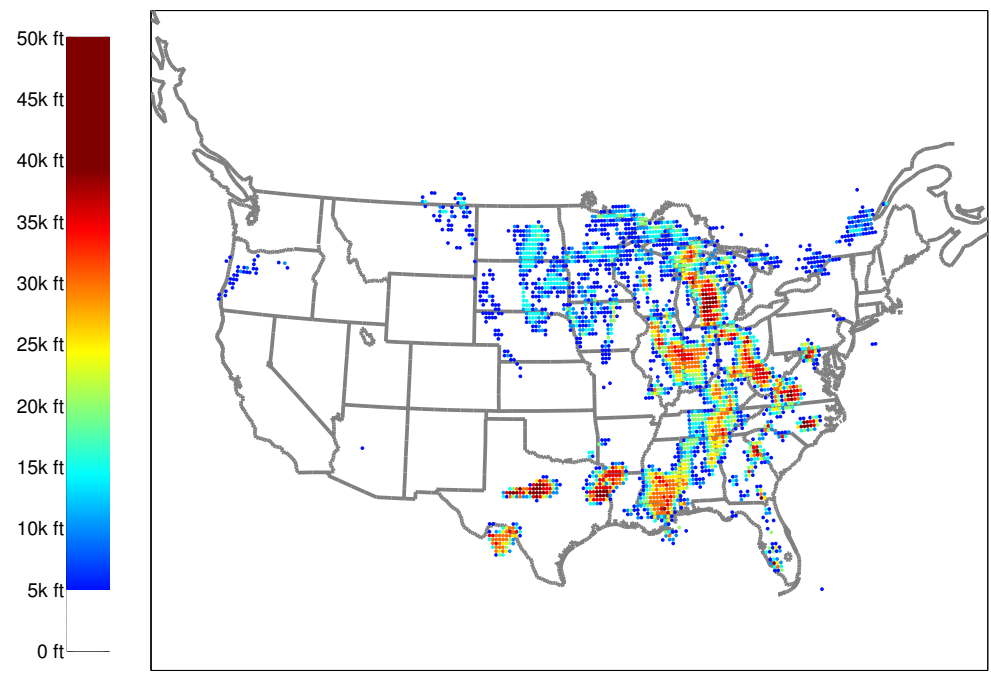




\section{Lower resolution weather data}

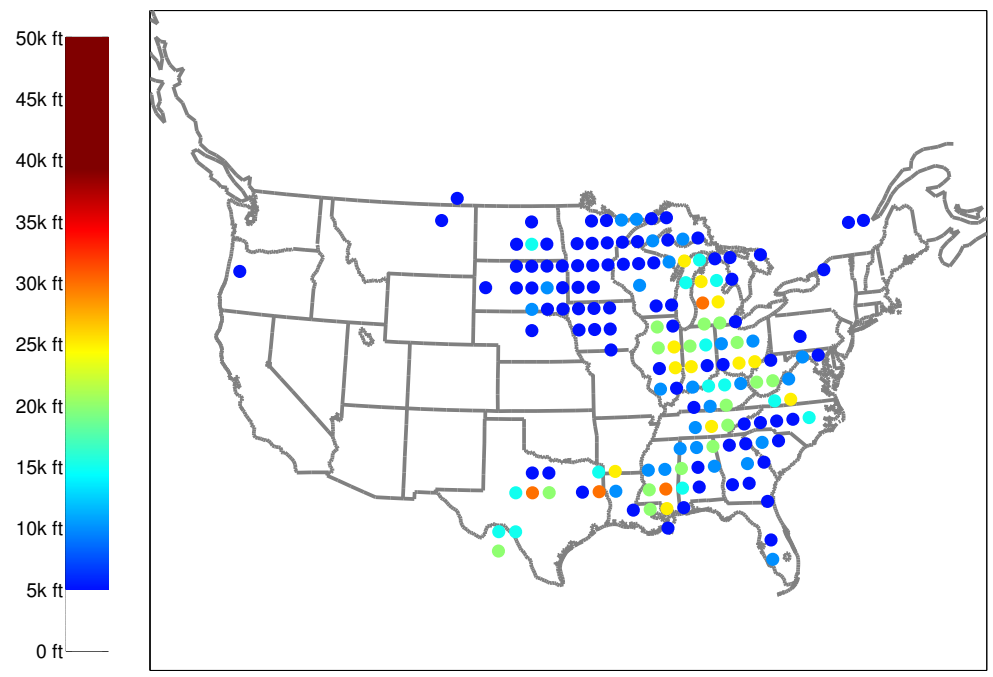




\section{Lower resolution weather data}

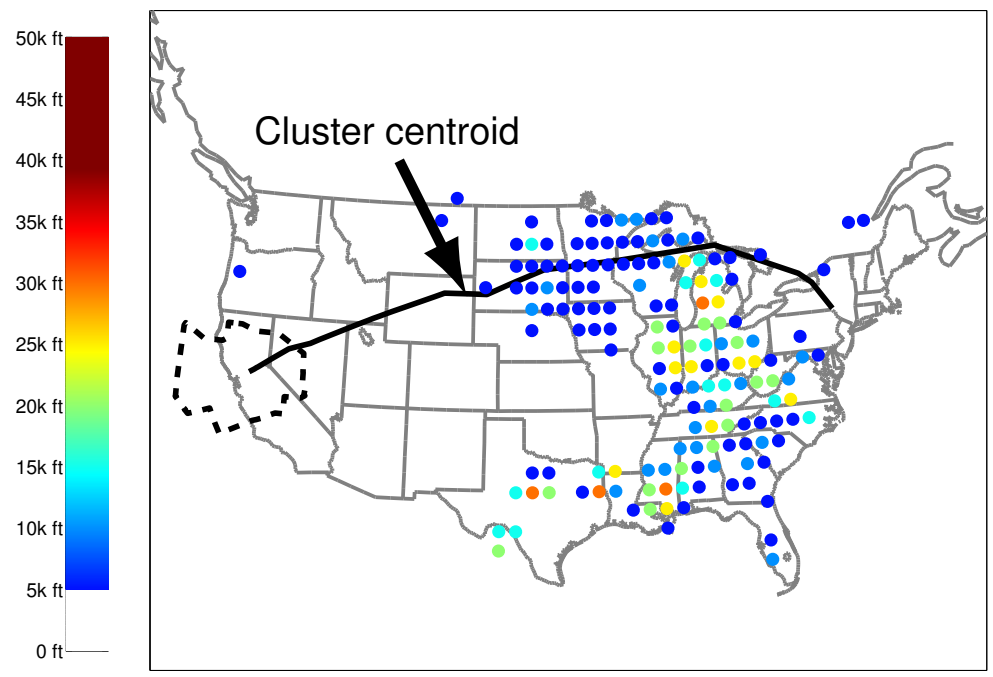




\section{Lower resolution weather data}

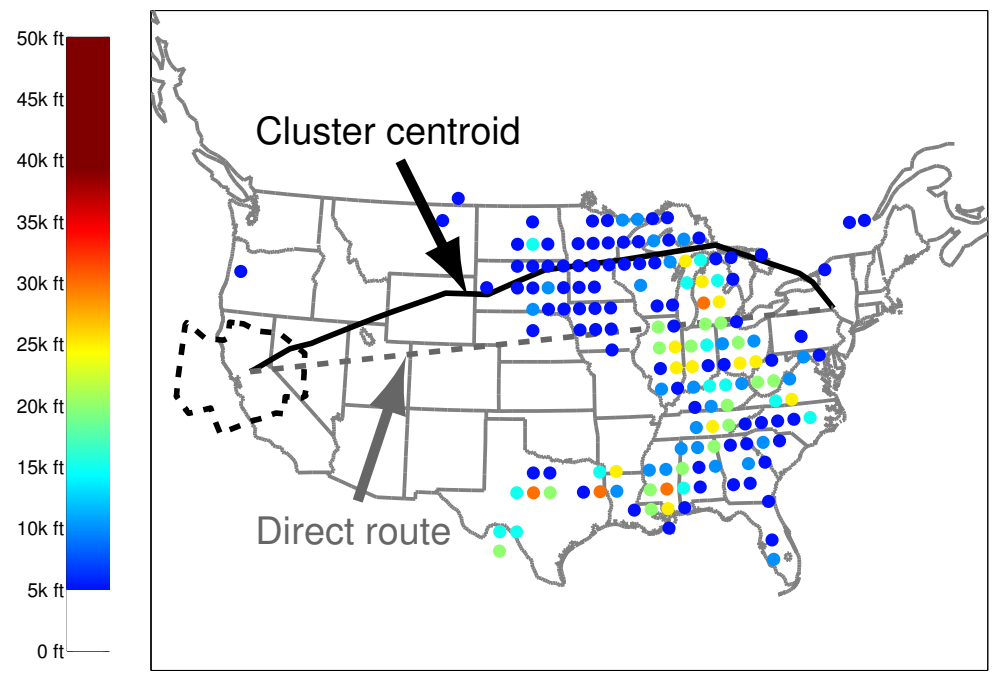




\section{Lower resolution weather data}

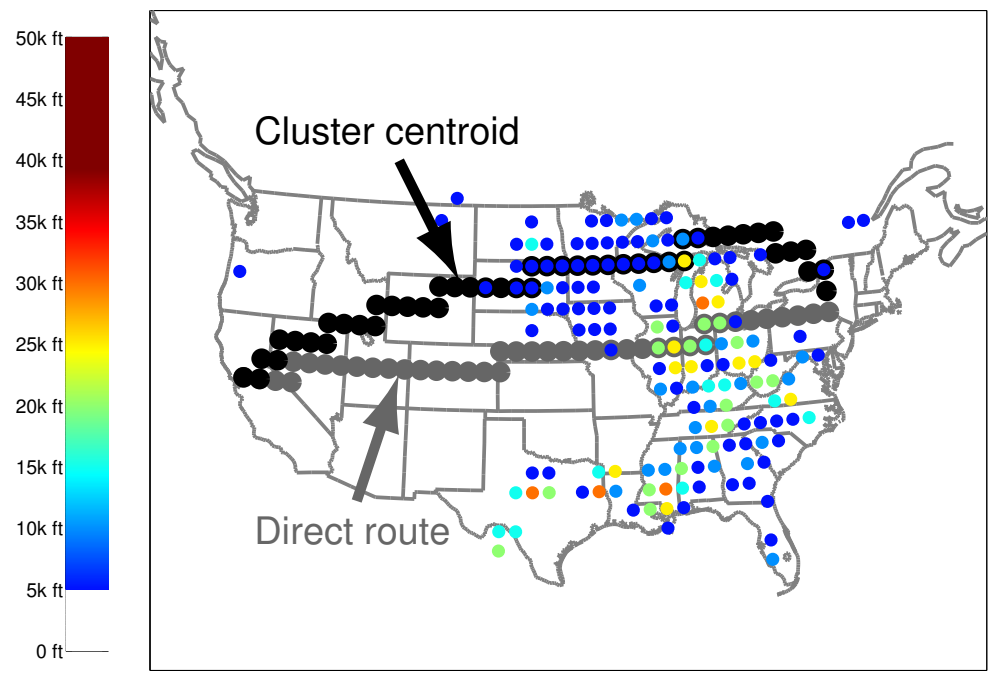




\section{Outline}

- Methodology

- Identification of routes used by flights

- Identification of similar routes

- Weather feature extraction

- Development of predictive models

- Prediction results

- Concluding remarks 
Reduced data

- June to August 2011

$\Rightarrow 2,208$ one-hour time windows

- $\quad 905$ ZNY-bound routes used

$\Rightarrow 253$ reroute clusters

$\Rightarrow 20$ most frequently used clusters

(used 50 to 240 times)

- 2,614,920 echo top data points per hour

$\Rightarrow \quad 1,000$ echo top points per hour

$\Rightarrow \quad 34$ created features per hour per cluster 
Reduced data

- June to August 2011

$\Rightarrow 2,208$ one-hour time windows

- $\quad 905$ ZNY-bound routes used

$\Rightarrow 253$ reroute clusters

$\Rightarrow 20$ most frequently used clusters

(used 50 to 240 times)

- 2,614,920 echo top data points per hour

$\Rightarrow \quad 1,000$ echo top points per hour

$\Rightarrow \quad 34$ created features per hour per cluster

Data for model development for one cluster

- 2,208 observations

- 34 created features

- class label

+ reroute cluster used

- reroute cluster not used 


\section{Model performance metrics}

\section{Classification error}

$$
\varepsilon=\frac{\# \text { incorrectly predicted observations }}{\text { total \# observations }}
$$




\section{Model performance metrics}

\section{Classification error}

$$
\varepsilon=\frac{\# \text { incorrectly predicted observations }}{\text { total \# observations }}
$$

True positive rate

TPR $=\frac{\# \text { of correctly predicted positive observations }}{\text { total \# of positive observations }}$ 


\section{Model performance metrics}

\section{Classification error}

$$
\varepsilon=\frac{\# \text { incorrectly predicted observations }}{\text { total \# observations }}
$$

True positive rate

TPR $=\frac{\# \text { of correctly predicted positive observations }}{\text { total \# of positive observations }}$

True negative rate

$\mathrm{TNR}=\frac{\# \text { of correctly predicted negative observations }}{\text { total } \# \text { of negative observations }}$ 


\section{Decision tree}

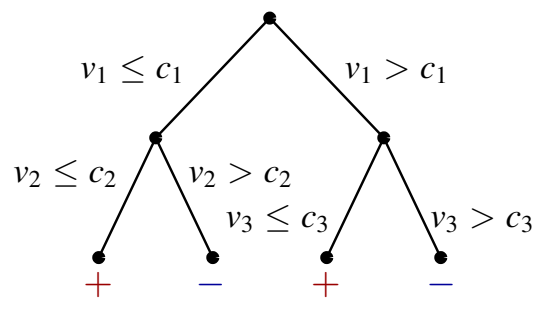




\section{Decision tree}

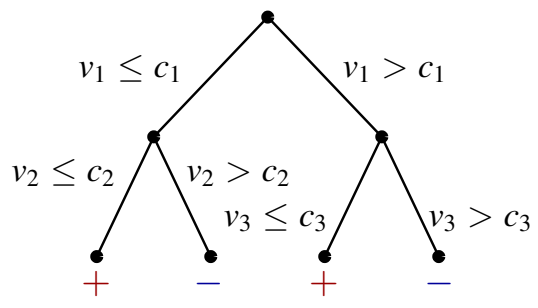

- Shallow trees cannot capture more complex connections

- Deep trees tend to overfit 


\section{Random forest}

- Consists of many weak learners (shallow decision trees)

- Each decision tree is built with:

- Randomly selected subset of observations

- Randomly selected subset of features

- Ensemble prediction: weighted vote of each weak learner 


\section{Random forest}

- Consists of many weak learners (shallow decision trees)

- Each decision tree is built with:

- Randomly selected subset of observations

- Randomly selected subset of features

- Ensemble prediction: weighted vote of each weak learner

$\Rightarrow$ Advantage: reduce sensitivity to noise $\Rightarrow$ reduce overfitting 


\section{Model development}

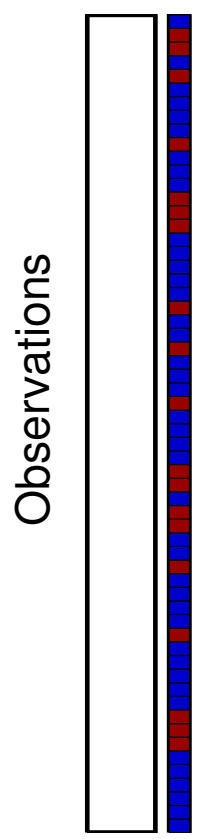




\section{Model development}

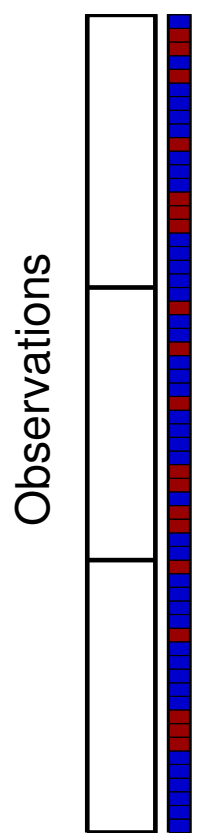




\section{Model development}

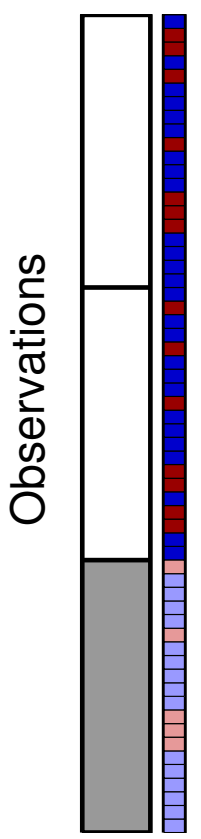




\section{Model development}

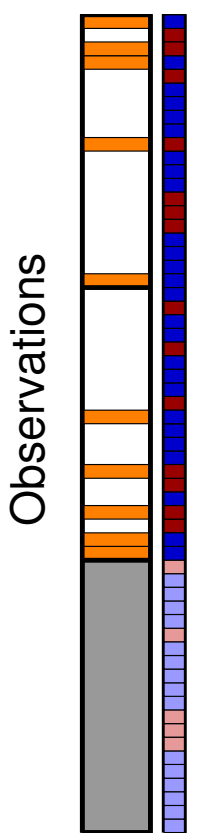




\section{Model development}

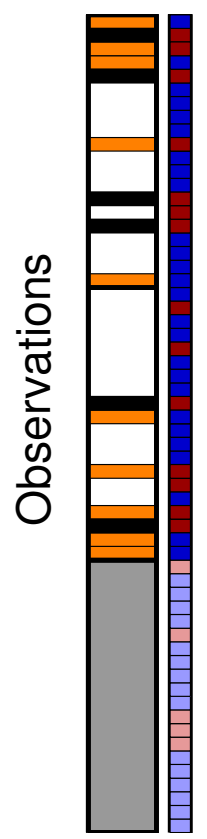




\section{Model development}

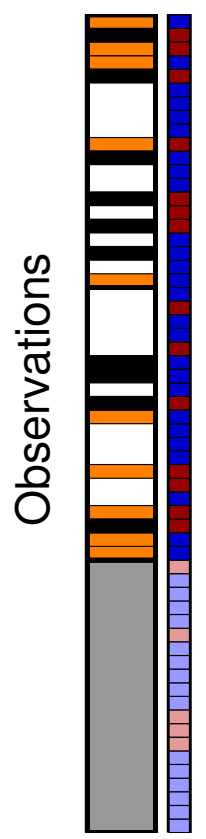




\section{Model development}

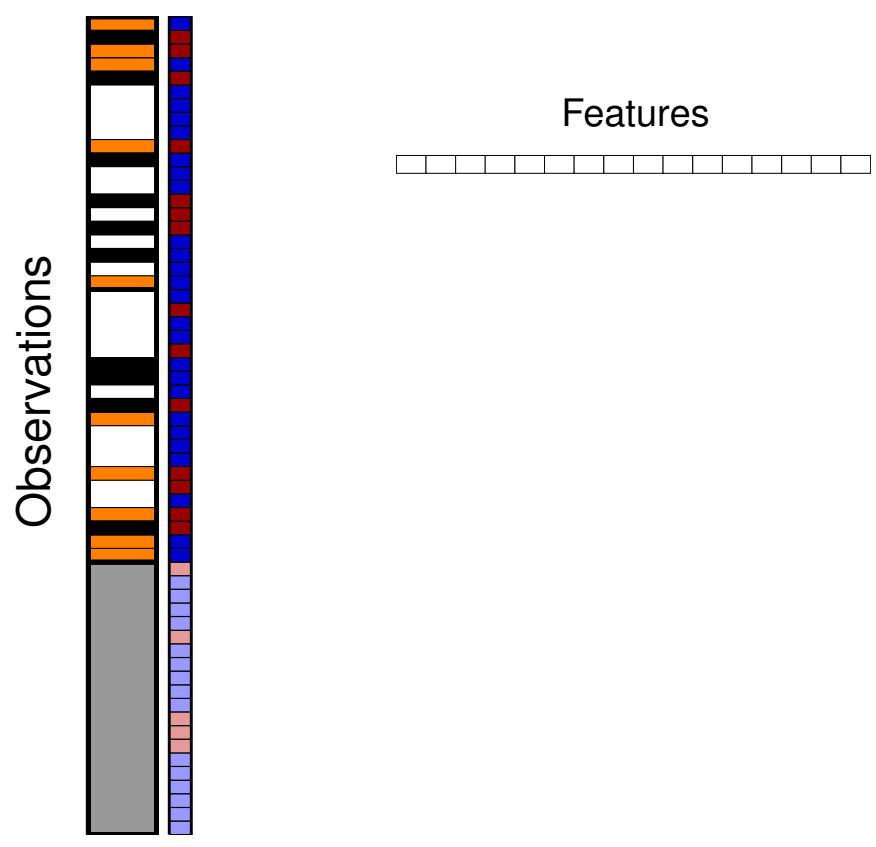




\section{Model development}

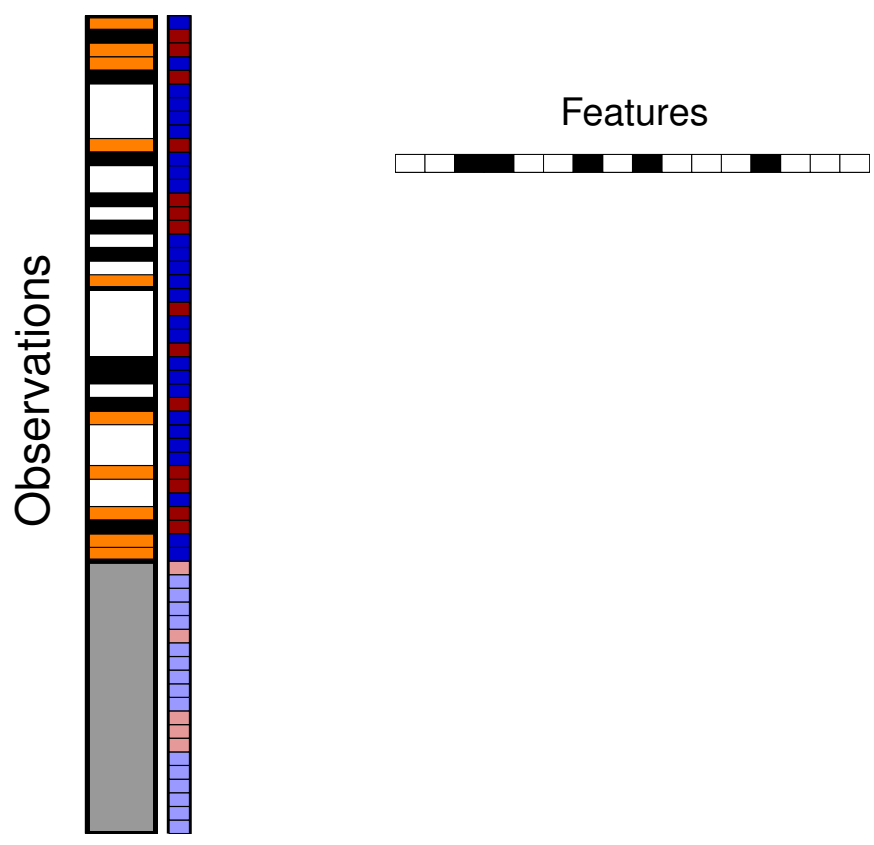




\section{Model development}

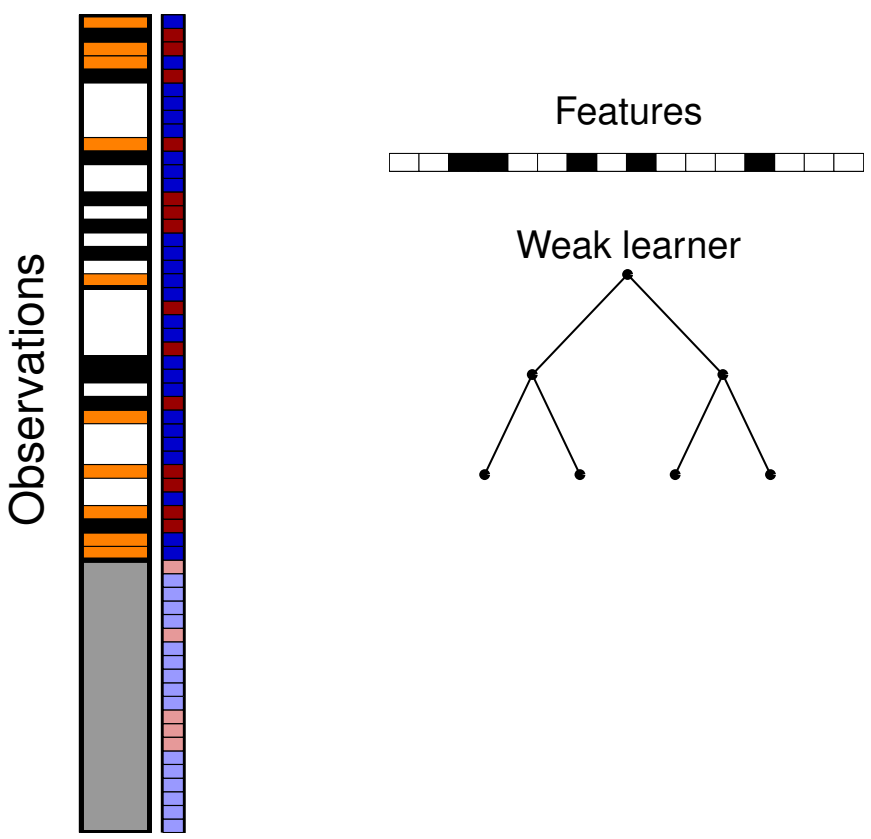




\section{Model development}

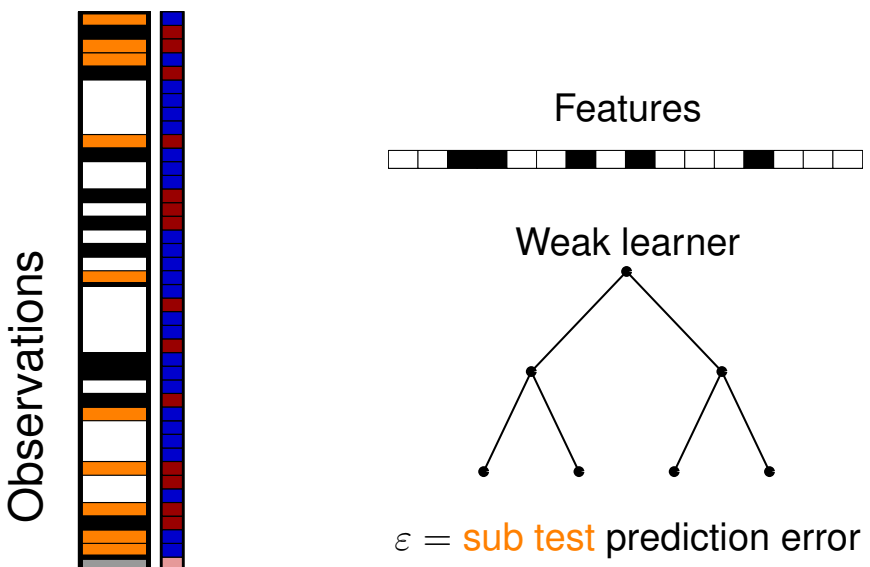




\section{Model development}

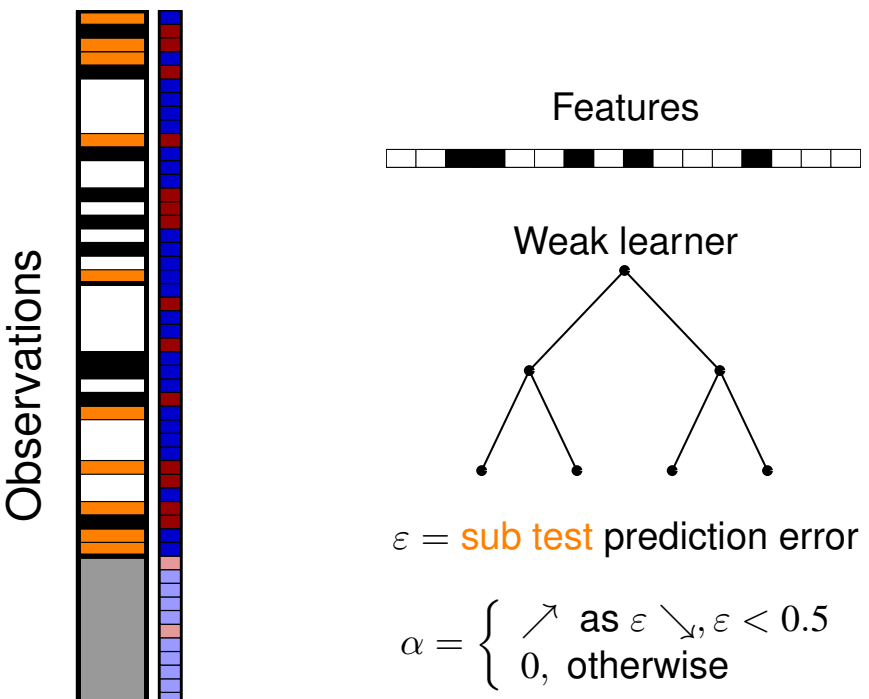




\section{Model development}

Weak learner 1

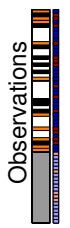

Weak learner 2

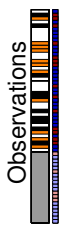

Features
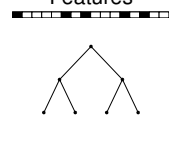

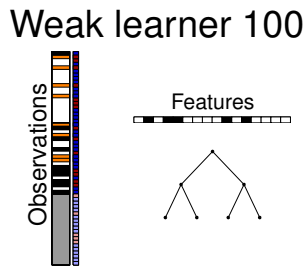




\section{Model development}

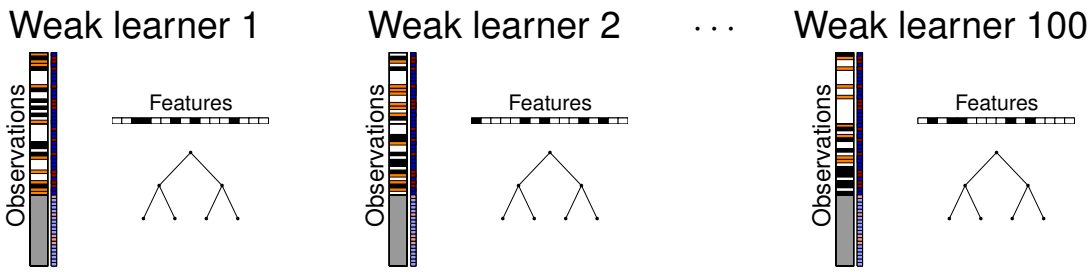

Ensemble prediction:

Weighted vote from each weak learner 


\section{Model development}

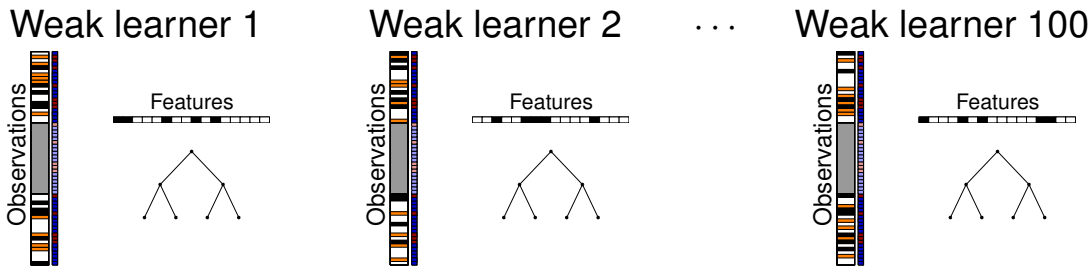

Ensemble prediction:

Weighted vote from each weak learner 


\section{Model development}

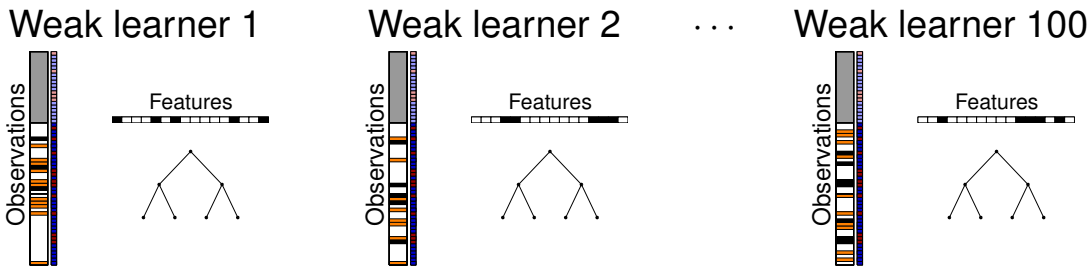

Ensemble prediction:

Weighted vote from each weak learner 


\section{Outline}

- Advisory details

- Methodology

- Identification of routes used by flights

- Identification of similar routes

- Weather feature extraction

- Development of predictive models

- Prediction results

- Concluding remarks 


\section{Prediction results}

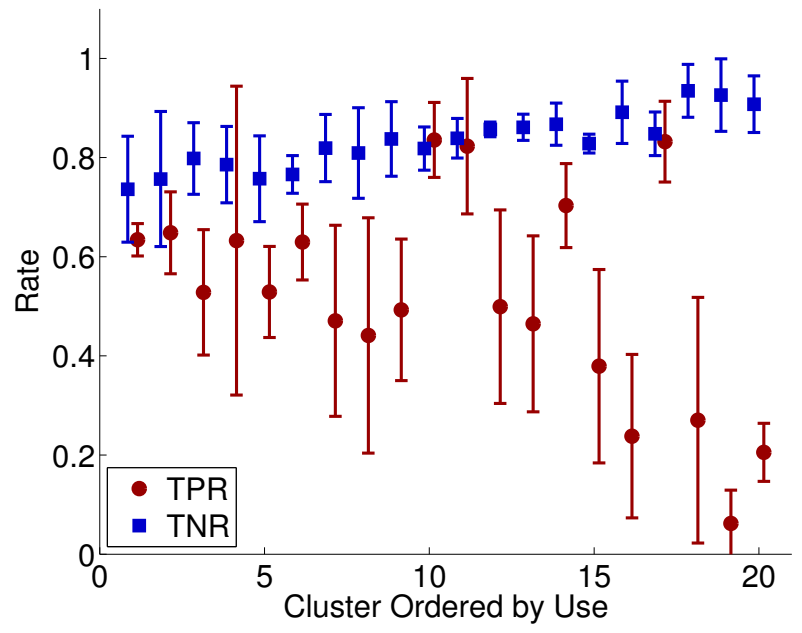




\section{Synthetic Minority Oversampling Technique (SMOTE)}

Within the training set:

- Select a positive observation

- Select one of its nearest neighbors

- Create a new observation:

Convex combination of these two observations 


\section{Prediction results with SMOTE}

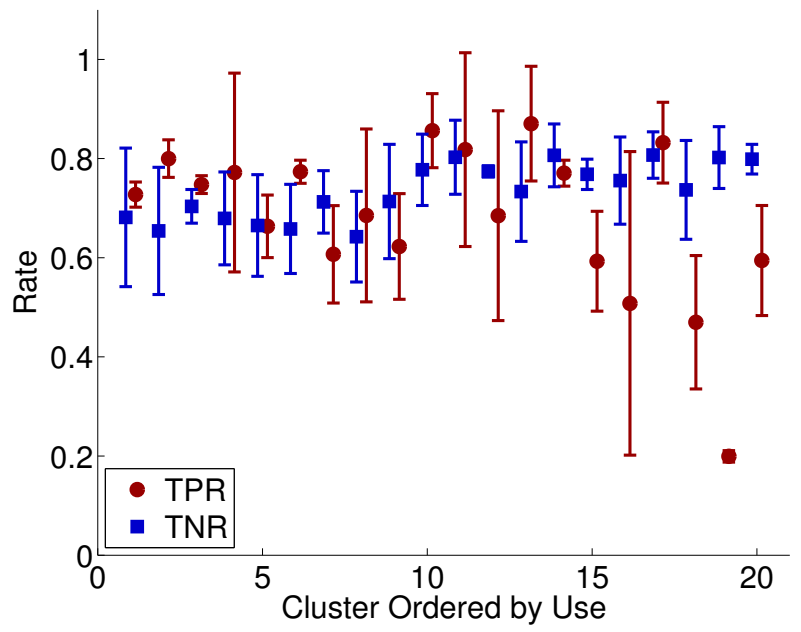




\section{Outline}

- Advisory details

- Methodology

- Identification of routes used by flights

- Identification of similar routes

- Weather feature extraction

- Development of predictive models

- Prediction results

- Concluding remarks 


\section{Conclusions and future work}

\section{Conclusions}

- Developed a framework to

- analyze the historical use of reroutes

- develop models to predict reroute use

- With improvements, this approach could provide insight into advisory use 


\section{Conclusions and future work}

\section{Conclusions}

- Developed a framework to

- analyze the historical use of reroutes

- develop models to predict reroute use

- With improvements, this approach could provide insight into advisory use

\section{Future work}

- Include weather conditions at fixes and along jet routes

- Use Convective Weather Avoidance Model (CWAM)

- Use Collaborative Convective Forecast Product (CCFP) 


\title{
Questions?
}

\author{
Heather Arneson \\ heather.arneson@nasa.gov
}

